\title{
The value of SHOX2 methylation test in peripheral blood samples used for the differential diagnosis of lung cancer and other lung disorders
}

\author{
M. KONECNY ${ }^{1, *}$, J. MARKUS ${ }^{1}$, I. WACZULIKOVA ${ }^{2}$, L. DOLESOVA ${ }^{3}$, R. KOZLOVA ${ }^{1}$, V. REPISKA ${ }^{3}$, H. NOVOSADOVA ${ }^{4}$, I. MAJER ${ }^{4}$ \\ ${ }^{1}$ Department of Clinical Genetics, St. Elizabeth Cancer Institute, Bratislava, Slovakia; ${ }^{2}$ Department of Nuclear Physics and Biophysics, Faculty of \\ Mathematics, Physics and Informatics, Comenius University, Bratislava, Slovakia; ${ }^{3}$ Institute of Medical Biology, Genetics and Clinical Genetics, \\ Faculty of Medicine and Faculty Hospital, Comenius University, Bratislava, Slovakia; ${ }^{4}$ Department of Pneumology and Phtiseology, University \\ Hospital, Bratislava, Slovakia
}

*Correspondence: michal.konecny@ousa.sk

Received April 19, 2015 / Accepted September 7, 2015

\begin{abstract}
Methylation of the cytosine residues within the CpG dinucleotides plays an important role in the fundamental cellular processes, human diseases and even cancer. The DNA methylation represents a very stable sign and therefore may be used as a valuable marker for cancer screening. Epigenetic cancer biomarkers are independent of classical morphology and thus show extensive potential to overcome the limitations of cytology. Several epigenetic cancer markers have been reported to be detectable in body fluids such as bronchial aspirate, sputum, plasma and serum.

Short stature homeobox gene 2 (SHOX2) encodes a homeo-domain transcription factor, which has been identified as a close homologue of the SHOX gene and both genes are involved in skeletogenesis and heart development. Methylation of SHOX2 gene has been shown to be present at high prevalence in carcinomas of lung, however may also be used to identify other tumour entities.

In the presented study, we have compared suitability of two types of material associated with lung cancer for the detection of SHOX2 methylation. We have confirmed that methylation of SHOX2 gene represents reliable marker of lung malignancies. The parallel tests in the blood plasma revealed that it may represent a good alternative material for testing of the SHOX2 methylation, making the test available to patients who are unable to undergo bronchoscopy.
\end{abstract}

Key words: DNA methylation test, SHOX2, lung cancer, bronchial lavage, blood plasma

Lung cancer represents the most common malignancy and the main cause of cancer-related death worldwide [1]. Onset of the lung cancer is the result of many factors, genetic, epigenetic and environmental ones. Majority of lung cancers, approximately $80-90 \%$, could be attributed to cigarette smoking [2]. Nowadays, there is sufficient evidence to infer a causal relationship between smoking and lung cancer. Smoking causes genetic changes in cells of the lung that ultimately lead to the development of lung cancer. In consequence, $85 \%$ of all lung cancer deaths are estimated to be attributed to cigarette smoking [3]. Nevertheless, about 10-20\% of lung cancer cases occur in non-smokers [4], what clearly points to the fact that lung cancer risk is not explainable simply by cigarette smoking. Thus, besides age and gender, other risk factors, such as environmental exposure, lung function, or genetic markers need to be taken into account [3]. In 2005, the mortality of lung cancer in Slovak men was estimated at 50.3 (out of 100 000) and in Slovak women 7.6 (out of 100 000) [5].

Based on histology, lung cancers can be divided in two main histological groups, non-small cell (85\%) and small cell lung cancer. This grouping has major impact on further clinical management of the patients, treatment and also on the prognosis of the disease [6]. One of the most important factors contributing to the successful and effective treatment is the early detection of the disease, which may have major impact on the outcome. However, imaging or cytology strategies often fail to detect early stages of lung cancer [7]. Lack of effective tools to diagnose lung cancer at an early stage (that means before it has spread to regional lymph pulmonary nodule or metastasized beyond the lung) results in a 5-year mortality rate from $80 \%$ to $85 \%$ [8]. Current efforts are focused on identification, selection and validation of new non-invasive or 
minimally invasive markers which might assist early diagnosis, prognosis of the defined disease and prediction of response to treatment.

Recent data support the view that genetic and epigenetic factors play an important causal role in carcinogenesis [9]. Most common events observed in the lung cancer are epigenetic alterations, such as promoter DNA methylation, which is often associated with gene silencing.

Epigenetic abnormalities are presented in all human cancers. Cancer cells globally present DNA hypomethylation or also hypermethylation of tumor suppressor genes [10]. Well-studied gene examples are hypermethylation of $p 16$ [11], H-cadherin, RASSF1A, APC and DAPK1 genes [12]. Compared to other molecular markers, such as mRNA or proteins, DNA methylation represents a chemically stable marker. It is a covalent DNA modification which can be easily detected by standard molecular biology methods and which thus might constitute such an early indicator of neoplastic transformation. This assumption is based on the fact of high (above average) level of p16 methylation observed in pulmonary hyperplasia (17\%), dysplasia (24\%) and lung carcinoma in situ (50\%) [13].

Information about abnormal DNA methylation can be useful in disease phenotyping, more precise histological typing, determining the tumor aggressiveness, prediction of therapy efficiency and even detection of risk of cancer development. Importantly, it can be detected in many different types of tissues and samples, including tumor tissues, cancer cells in body fluids, cell-free tumor DNA in blood plasma. Lung cancer-associated aberrant DNA methylation is often detected in patient's sputum [14], bronchoalveolar lavage [15] or saliva [16].

SHOX2 gene (short stature homeobox 2 gene) is localized on the $3 \mathrm{rd}$ chromosome (3q25.32), is $10 \mathrm{~kb}$ in size and comprises of 7 exons encoding a 319 amino-acid protein. It is a member of the homeobox genes family that encodes proteins with specific 60 -amino acid DNA binding domain. Two splicing variants of SHOX2 transcripts are known: A (993 bp) and B (570 bp) [17]. Two huge CpG islands are located within the SHOX2 gene, one covering $1 \mathrm{~kb}$ at the $5^{\prime}$ end, another 500bp island is located at the $3^{\prime}$ end. The homeobox genes have been characterized as transcription factors involved in pattern formation in invertebrates as well as vertebrates. SHOX2 has been shown to play a major role during skeletogenesis and heart development [18]. The SHOX2 gene is exclusively expressed in the anterior mesenchyme region of the developing secondary palate, with the highest expression during the early stage of palate development [19]. Generally, the homeobox genes represent a pseudoautosomal locus that is thought to be responsible for idiopathic short stature, and for short stature phenotype of Turner syndrome patients.

The SHOX2 genes, together with SEPT9, are the methylation markers used in routine diagnostics of lung or colorectal cancer respectively. Both markers have been validated on large numbers of patients and have been reported to have high spe- cificity and sensitivity. Aberrant DNA methylation of SHOX2 is a hallmark of lung tumors and testing the methylation has been validated in bronchial lavage samples from patients with suspected lung cancer, including those with negative cytopathological result and those with no visual detection of the tumor [20].

Kneip et al. [21] have reported that lung cancer-associated DNA methylation of SHOX2 gene can also be successfully detected in blood plasma with sensitivity of $60 \%$ and specificity of $90 \%$. Begum et al. [22] tested the methylation status of 6 different genes (APC, CDH1, MGMT, DCC, RASSF1A, AIM1). Their results suggest $100 \%$ specificity of $D C C$ gene methylation and they were able to correctly identify all lung cancer patients using this method. However, these results need to be confirmed in longitudinal studies.

Since SHOX2 methylation in blood plasma was previously reported as sensitive and specific diagnostic test, it is reasonable to consider and explore in more detail the benefits and limitations of this noninvasive alternative to the current bronchial lavage test [21]. Actually, CE marked IVD molecular test based on the SHOX2 DNA methylation in lavage samples is commercially available in Europe [20]. On the other hand it is still not clear if the blood plasma SHOX2 methylation test may completely substitute the lavage testing in the diagnostics and even if the presence of SHOX2 methylation in blood plasma sufficiently reflects the presence of tumor. The focus of the study was to evaluate evidence for using of blood plasma methylation method in diagnosing patients with lung cancer, either as a replacement for or an addition to currently used bronchial lavage.

The main objective of our study was to determine the diagnostic performance of SHOX2 methylation tests in parallel samples of bronchial lavage and peripheral blood from the same patient. The secondary objective was to evaluate the degree of agreement between these two methods.

\section{Materials and methods}

Samples. We can define our study as a type of observational case-control study with data collected in a cross-sectional manner. The cases (positive group) and controls (negative group) were selected according to the presence or absence of malignant disease defined by cytology or histology analysis.

In our study, we recruited patients undergoing bronchoscopy at the Department of Pneumology and Phtiseology of University Hospital in Bratislava for suspicion of lung cancer or alternate non-cancer disease. From all eligible patients parallel samples of bronchial lavage and peripheral blood were obtained. Bronchial lavages were collected into Saccomano fixative in the ratio $1: 1$, specifically $1 \mathrm{ml}$ of lavage was mixed with $1 \mathrm{ml}$ of fixative, and stored at $4^{\circ} \mathrm{C}$ until further use. Peripheral blood was collected in the volume of $7 \mathrm{ml}$ into EDTA tubes and centrifuged. Plasma was collected and stored at $-20^{\circ} \mathrm{C}$ until analysis (no more than 2 weeks). Totally, 69 parallel samples were collected at Department of Clinical Genetics, St. Elizabeth 
Cancer Institute between January 2014 and December 2014, with addition time for data collection in January and February of 2015. Based on the cytological or histological analysis, 38 samples were positive for malignant lung disease, the rest of the samples $(\mathrm{n}=31)$ were considered negative for malignancy. These patients underwent bronchoscopy and lavage due to other non-malignant lung condition and no malignancy was found in their samples.

DNA isolation. DNA was isolated using commercial Epi proLung BL DNA preparation kit (Epigenomics) for bronchial lavages and Epi proColon Plasma Quick kit (Epigenomics) for peripheral blood samples according to manufacturer's recommendations. Bisulfide conversion of purified DNA was performed by addition of bisulfite reagent and denaturation buffer, at $85^{\circ} \mathrm{C}$ for 45 minutes in thermo-shaker at 1000 $\mathrm{rpm}$. Treated DNA was stored at $4^{\circ} \mathrm{C}$ and analyzed within 24 hours.

Real time PCR analysis. The real-time PCR analysis was performed using the Epi proLung BL real-time PCR kit (Epigenomics) on the ABI 7500 Fast real-time PCR instrument (Life Technologies) using specific temperature profile $\left(95^{\circ} \mathrm{C} / 15\right.$ min., 40 cycles of $56^{\circ} \mathrm{C} / 30$ sec., $95^{\circ} \mathrm{C} / 15 \mathrm{sec}$.). Sequences of the probes and primers were previously published [23]. The PCR analysis uses two target-specific probes; one for methylationprone sequence of $S H O X 2$ gene labeled with FAM and second targeting methylation-independent sequence in $A C T B$ gene labeled with JOE. The PCR was performed in two parallel reactions for each sample. Calibrator and positive/negative control samples were analyzed in each run as well (Control Work-flow kit, Epigenomics). Threshold and baseline settings were set to 0.01 , baseline start at 3 and baseline stop at 15 for $A C T B$ and 20 for SHOX2. The run was considered valid if the cycle threshold $\left(\mathrm{C}_{\mathrm{T}}\right)$ value for $A C T B$ in positive sample was below 31 and $\mathrm{C}_{\mathrm{T}}$ for $S H O X 2$ was below 37 ; the $\mathrm{C}_{\mathrm{T}}$ for $A C T B$ in negative control was between 28-37 and $\mathrm{C}_{\mathrm{T}}$ for $S H O X 2$ above 37. Lavage samples were considered positive if $\mathrm{C}_{\mathrm{T}}$ for $A C T B$ was below 29 and blood samples were considered positive if $\mathrm{C}_{\mathrm{T}}$ for $A C T B$ was below 35. Calibrator sample was considered positive if $\mathrm{C}_{\mathrm{T}}$ for $A C T B$ was below 32 . Methylation data analysis was performed using $\Delta \Delta \mathrm{C}_{\mathrm{T}}$ method according to the Epi proLung $\mathrm{BL}$ kit manual. In the first step $\Delta \mathrm{C}_{\mathrm{T}}$ for each sample as well as calibrator was calculated using the following equation: $\mathrm{C}_{\mathrm{T}}(S H O X 2)-\mathrm{C}_{\mathrm{T}}(A C T B)$. The $\Delta \Delta \mathrm{Ct}$ value was calculated by subtracting $\Delta \mathrm{C}_{\mathrm{T}}$ of each sample from $\Delta \mathrm{C}_{\mathrm{T}}$ of calibrator. If the $\Delta \Delta \mathrm{C}_{\mathrm{T}}$ value was lower than 9.5 , samples were considered positive for methylated SHOX2.

Statistical analysis. The diagnosis of lung cancer (selection of cases) was based on biopsy/cytology results. Descriptive and bivariate statistics were performed on all patients' characteristics. Both groups were generally balanced with regard to gender $(\mathrm{P}>0.05)$ and unbalanced with regard to the age distribution $(\mathrm{P}<0.05)$. Agreement analysis was used to evaluate the degree of agreement and disagreement of both methods over categories in $2 \times 2$ tables. Diagnostic performance of each single method was evaluated using the biopsy/cytology results.
Cohen's Kappa, Maxwell's chi-square and McNemar's statistics were used to test for agreement, disagreement and significant differences. In broad terms a Kappa below 0.2 indicates poor agreement and a Kappa above 0.8 indicates very good agreement beyond chance. Maxwell's chi-square statistic tests for overall disagreement between the two methods' readings. The general McNemar statistic tests for asymmetry in the distribution of subjects about which the readings disagree, i.e. disagreement more over some categories of response than others.

Statistical modeling. We used multivariable logistic regression for construction and evaluation of lung cancer risk models. The available clinical variables included age, and the following dichotomous variables: gender ( 1 male, 0 female), smoking status ( 1 smoker/former smoker, 0 non-smoker), cell type (1 SCLC, 0 otherwise, i.e. NSCLC all subtypes). The model analysis and selection was made using goodness of fit tests (Pearson, deviance and Hosmer-Lemeshow tests) and Akaike's information criterion. Because of different age distribution between cases and controls all tested models were adjusted to age. Effect size for significant or clinically important explanatory variables was estimated using odds ratio (OR) and a corresponding 95\% confidence interval (95\% CI). Receiver Operating Characteristic (ROC) curve analysis was used to define detection cut-off points for analyzed diagnostic tests represented by the multivariable models. In a ROC curve sensitivity (probability of positive test when disease is present) is plotted against 1-specificity (probability of negative test when disease is absent). Resulting ROC curves were compared using AUC criterion (the area under the curve). The accuracy, sensitivity, specificity, positive predictive value (PPV, i.e. post-test likelihood of disease), and negative predictive value (NPV, i.e. post-test likelihood of of no disease), all along with their 95\% CI, were also calculated for the bronchial lavage and peripheral blood tests, as well as for the models. For each of the models, patients that had a probability of lung cancer $\geq 0.5$ were classified as having lung cancer, and patients with a probability $<0.5$ were classified as not having lung cancer. The final model was composed of four explanatory variables that most contributed to the risk.

All tests were conducted at significance level of 5\% using StatsDirect 2.8.0 software.

\section{Results}

Overall methylation analysis. Samples were collected from 69 patients, specifically 49 men and 20 women. Average age of the patients was 60.4 years of age; 54.1 years for patients with diagnoses other than lung cancer (further referred as negative) and 66.7 years for patients with confirmed lung cancer. There were 16 non-smokers (23.2\%), 3 in positive group and 13 in negative group. The rest of the patients (76.8\%) were active or former smokers ( $\mathrm{n}=53), 35$ with detected malignancy and 18 without lung cancer (Table 1). Two types of material were 
Table 1. Distribution of the tested group of samples according to selected categories and based on tumor presence.

\begin{tabular}{|c|c|c|c|c|c|c|c|c|c|c|}
\hline \multirow[t]{2}{*}{ Category } & \multirow[t]{2}{*}{ Samples } & \multicolumn{5}{|c|}{ Tumor samples } & \multirow{2}{*}{$\begin{array}{c}\text { P-value } \\
\text { Fisher } \\
\text { exact test }\end{array}$} & \multirow{2}{*}{$\begin{array}{c}\text { Tumor } \\
\text { samples } \\
\text { total }\end{array}$} & \multirow{2}{*}{$\begin{array}{l}\text { Non-tumor } \\
\text { samples } \\
\text { total }\end{array}$} & \multirow{2}{*}{$\begin{array}{c}\text { P-value } \\
\text { Fisher exact } \\
\text { test }\end{array}$} \\
\hline & & Stage I & $\begin{array}{l}\text { Stage II/ } \\
\text { LimDis }\end{array}$ & stage III & $\begin{array}{l}\text { stage IV/ } \\
\text { ExDis }\end{array}$ & $\begin{array}{c}\text { Undetermined } \\
\text { staging }\end{array}$ & & & & \\
\hline Total & & 1 & 4 & 8 & 22 & 3 & n.a. & 38 & 31 & n.a. \\
\hline \multirow{2}{*}{ Type of test } & Histology & 1 & 2 & 4 & 9 & 0 & n.a. & 16 & 0 & n.a. \\
\hline & Cytology & 0 & 2 & 4 & 13 & 0 & n.a. & 19 & 31 & n.a. \\
\hline \multirow{2}{*}{ Histological type } & NSCLC & 1 & 2 & 8 & 15 & 2 & & 28 & 0 & \\
\hline & SCLC & 0 & 2 & 0 & 7 & 1 & 0.585 & 10 & 0 & n.a. \\
\hline \multirow{2}{*}{ mSHOX 2 status * } & mSHOX2+ & 1 & 4 & 7 & 20 & 3 & & 35 & 9 & \\
\hline & mSHOX2- & 0 & 0 & 1 & 2 & 0 & $>0.999$ & 3 & 22 & $<0.0001$ \\
\hline \multirow{3}{*}{ Smoking status ${ }^{* *}$} & Non-smokers & 0 & 1 & 2 & 0 & 0 & & 3 & 13 & \\
\hline & Smokers & 1 & 3 & 6 & 18 & 2 & 0.379 & 30 & 14 & 0.0013 \\
\hline & Former smokers & 0 & 0 & 0 & 4 & 1 & & 5 & 4 & \\
\hline
\end{tabular}

Abbreviations: n.a. not applicable; LimDis - limited disease of SCLC, linked to Stage II; ExDis - extended disease of SCLC, linked to Stage IV. Due to low counts in these categories, statistical analysis in tumor samples was performed on $2 \times 2$ contingency tables for pooled data (stages I+II and III+IV). NSCLC - non-small cell lung cancer, SCLC - small cell lung cancer, mSHOX2+/- - positive/negative methylation of SHOX2.

${ }^{*}$ Sample was considered positive for SHOX2 methylation if positivity in at least one type of material was detected

** Analysis was performed on non-smokers vs. smokers or former smokers.

collected from each patient: bronchial lavage and peripheral blood. Taken together we analyzed 138 samples in duplex real-time PCR reactions in parallels of plasma- and lavageisolated DNA. The overall frequency of SHOX2 methylation in analyzed set was estimated at $63.8 \%$ (44 out of 69).

Methylation of SHOX2 gene was detected in 26 samples, specifically in 20 tumor samples and 6 negative samples. Unmethylated samples were represented by 33 specimens, 11 samples in group with diagnosed tumor and 22 in negative group. Results from 10 samples were considered invalid (Table 2). Out of 38 histologically positive patients, the analysis of SHOX 2 was valid in 37 bronchial lavage samples and 31 blood samples. The SHOX 2 methylation was detected in 31 of 37 valid bronchial lavage samples and 20 of 31 blood samples. On the other hand, the SHOX2 was found unmethylated in 22 of 26 valid bronchial lavage samples from cancer-free patients and in 22 of 28 valid blood samples.

Presence of methylation in positive and negative group. Four different histological subtypes of lung cancer were distinguished in the positive sample group: squamous cell carcinomas, adenocarcinomas, small cell carcinomas, and large cell carcinomas. SHOX2 methylation was detected in almost all cancer samples, however 3 adenocarcinomas were negative (Table 3).

For 31 patients of no-malignancy (negative) group, the following diagnoses were determined: undetermined diagnosis $(n=6)$, bronchopneumonia $(n=7)$, sarcoidosis $(n=7)$, lymphomas $(n=4)$, tuberculosis $(n=3)$, mesothelioma $(n=1)$, cancer metastasis of non-lung origin $(n=1)$, pulmonary nodule $(n=1)$, foreign body $(n=1)$. The highest percentage of SHOX2 methylated samples was found among lymphomas $(50 \%)$. Also, in sample of mesothelioma, non-lung tumor metastasis and pulmonary nodule $(\mathrm{n}=3)$, SHOX2 methylation was detected. On the other hand, methylation was not detected in any of TBC samples, and only in $14.3 \%$ of bronchopneumonia and also sarcoidosis samples.

Detailed pairwise comparison of patients, in which analysis from both types of material was valid, revealed that in the histologically positive group the result of SHOX2 test correlated in 17 samples and was discordant in 13 samples. In the histologically negative group, the results of both tests correlated in 16 cases and were discordant in 7 cases. No significant

Table 2. Results of the SHOX2 methylation analysis linked to the type of the analyzed material.

\begin{tabular}{lccc}
\hline Tumor samples $(\mathbf{n}=\mathbf{3 8})$ & Blood mSHOX2 INVALID & Blood mSHOX2- & Blood mSHOX2+ \\
\hline Lavage mSHOX2 INVALID & 0 & 0 & 1 \\
Lavage mSHOX2- & 2 & 1 & 3 \\
Lavage mSHOX2+ & 5 & 10 & 16 \\
\hline Non-tumor samples (n=31) & Blood mSHOX2 INVALID & Blood mSHOX2- & Blood mSHOX2+ \\
\hline Lavage mSHOX2 INVALID & 0 & 4 & 1 \\
Lavage mSHOX2- & 3 & 15 & 4 \\
Lavage mSHOX2+ & 0 & 3 & 1 \\
\hline
\end{tabular}

Subjects are stratified according to the clinical status. Data within each stratum are paired according to the subjects and classified into the categories based on the type of sample and methylations status. Agreement and disagreement analysis of methylation is in detail presented within the Results section. 
Table 3. Histological subtypes of positive samples divided according to the type of carcinoma.

\begin{tabular}{|c|c|c|c|c|c|}
\hline \multirow[t]{2}{*}{ Samples } & \multicolumn{3}{|c|}{ NSCLC } & \multirow[t]{2}{*}{ SCLC } & \multirow[t]{2}{*}{ Total } \\
\hline & SCC & $\mathrm{ADC}^{\#}$ & LCC & & \\
\hline mSHOX2 + & 19 & 6 & 1 & 9 & 35 \\
\hline$\%$ of row & $54.29 \%$ & $17.14 \%$ & $2.86 \%$ & $25.71 \%$ & \\
\hline$\%$ of col & $100 \%$ & $66.67 \%$ & $100 \%$ & $100 \%$ & $92.11 \%$ \\
\hline mSHOX2 - & 0 & 3 & 0 & 0 & 3 \\
\hline$\%$ of row & $0 \%$ & $100 \%$ & $0 \%$ & $0 \%$ & \\
\hline$\%$ of col & $0 \%$ & $33.33 \%$ & $0 \%$ & $0 \%$ & $7.89 \%$ \\
\hline Total & 19 & 9 & 1 & 9 & 38 \\
\hline$\%$ of $n$ & $50 \%$ & $23.68 \%$ & $2.63 \%$ & $23.68 \%$ & \\
\hline $\begin{array}{l}{ }^{*} P=0.0381(F \\
\text { Abbreviations: } \\
\text { cinoma, ADC } \\
\text { cell lung cance } \\
{ }^{*} \text { sample was } c \\
\text { one type of mo }\end{array}$ & $\begin{array}{l}\text {-Freemar } \\
\text { CLC - no } \\
\text { denocarci } \\
\text { dered posi }\end{array}$ & $\begin{array}{l}\text { Halton exa } \\
\text { small cell } 1 \\
\text { ma, LCC } \\
\text { e for } \mathrm{SHO}\end{array}$ & $\begin{array}{l}\text { test) } \\
\text { g cance } \\
\text { arge ce } \\
\text { methy }\end{array}$ & $\begin{array}{l}\text { C-squa } \\
\text { cinoma, } \\
\text { if positi }\end{array}$ & $\begin{array}{l}\text { ous cell car } \\
\text { LC - smal } \\
y \text { in at leas }\end{array}$ \\
\hline
\end{tabular}

asymmetry of disagreement in the subgroups was revealed. The overall agreement in a total of 53 samples was $62.26 \%$ (Kappa $=0.255 ; \mathrm{P}=0.029)$. Asymmetry of disagreement was not significant $(\mathrm{P}=0.18)$.

Agreement analysis of methylation in different material. Agreement between definite diagnosis and either bronchial lavage or peripheral blood test on SHOX2 methylation were above the lowest acceptable level of $70 \%$ of all classifications.

1. The bronchial lavage test results agreed in $84.13 \%$ (Kappa $=0.68,0.49$ to $0.86 ; \mathrm{P}<0.0001$ ) with no significant asymmetry of disagreement $(\mathrm{P}=0.53), \mathrm{PPV}=88.57 \%$ $(73.26 \%$ to $96.8 \%)$, change $=30 \%, \mathrm{NPV}=78.57 \%(59.05 \%$ to $91.7 \%)$, change $=38 \%$. Predictive value $(\mathrm{PV})$ despite negative test was $21.43 \%$ ( $8.3 \%$ to $40.95 \%)$, change $=-38 \%$, sensitivity $=83.78 \%$ (67.99 to 93.81$)$, and specificity $=84.62 \%(65.13$ to 95.65$)$.
2. The peripheral blood test results agreed in $71.19 \%$ (Kappa $=0.43,0.20$ to $0.65 ; \mathrm{P}=0.0004)$ with no significant asymmetry of disagreement $(\mathrm{P}=0.22), \mathrm{PPV}=76.92 \%(56.35 \%$ to $91.03 \%)$, change $=24 \%, \mathrm{NPV}=66.67 \%(48.17 \%$ to $82.04 \%)$, change $=20 \%$, PV despite negative test was $33.33 \%(17.96 \%$ to $51.83 \%)$, change $=-20 \%$, sensitivity $=64.52(45.37$ to 80.77$)$, and specificity $=78.57$ (59.05 to 91.71 ).

The independent contribution of each SHOX2 methylation test to the prediction of malignancy was evaluated in multivariable models adjusted for age, gender, and smoking status (Table 4). ROC curve analysis was then used to define the optimal cut-off point for classification. In a ROC curve the sensitivity (probability of +ve result when malignancy is present) is plotted against 1-specificity (probability of +ve test when malignancy is absent). Resulting ROC curves for the models constructed using either peripheral blood test or bronchial lavage test were compared using the AUC criterion (the area under the curve). Since the prediction model involving bronchial lavage test results was not essentially better than that using the peripheral blood test results as seen from their AUC values, here we present the ROC curve for the latter model only (Figure 1).

Diagnostic performance of the model constructed with biomarker SHOX2 methylation determined from the bronchial lavage samples at probability cut-off $\geq 0.5$ : sensitivity $=$ $89.19 \%$, specificity $=84.62 \%, \mathrm{PPV}=89.19 \%, \mathrm{NPV}=84.62 \%$, PV despite negative test $=15.38 \%$, correctly classified $=$ $87.3 \%$, likelihood ratio: LR of positive test $=5.797$ (2.629 to $14.582)$, LR of negative test $=0.128(0,050$ to 0,299$)$, AUC $=88.93 \%$.

The model constructed with biomarker SHOX2 methylation determined from the peripheral blood samples yielded quite similar results: sensitivity $=80.65 \%$, specificity $=78.57 \%, \mathrm{PPV}=80.65 \%, \mathrm{NPV}=78.57 \%$, PV despite negative test $=21.43 \%$, correctly classified $=79.66 \%$, likelihood ratio: LR of positive test $=3.763$ (1.966 to 8.024$)$, LR negative test $=0.246334$ (0.115 to 0.483$), \mathrm{AUC}=86.81 \%$ (Figure 1$)$.

Table 4. Logistic regression models fitted on patients' data.

\begin{tabular}{|c|c|c|c|c|c|c|}
\hline Model & $\begin{array}{c}\text { Deviance (goodness of fit) } \\
\text { P-value }\end{array}$ & $\begin{array}{c}\text { Deviance (likelihood ratio) } \\
\text { P-value }\end{array}$ & Coefficient & OR & $95 \% \mathrm{CI}$ & P-value \\
\hline Lavage test & 0.6500 & $<0.0001$ & n.a. & n.a. & n.a. & n.a. \\
\hline Intercept & n.a. & n.a. & -4.833 & n.a. & n.a. & $\mathrm{P}=0.0338$ \\
\hline Gender & n.a. & n.a. & -1.262 & 0.283 & (0.045 to 1.779$)$ & $\mathrm{P}=0.1784$ \\
\hline Age & n.a. & n.a. & 0.038 & 1.039 & (0.976 to 1.105$)$ & $\mathrm{P}=0.2313$ \\
\hline Smoking & n.a. & n.a. & 2.677 & 14.553 & (1.084 to 195.300$)$ & $\mathrm{P}=0.0433$ \\
\hline $\mathrm{mSHOX} 2$ & n.a. & n.a. & 3.011 & 20.314 & (4.125 to 100.000$)$ & $\mathrm{P}=0.0002$ \\
\hline Blood test & 0.7800 & $<0.0001$ & n.a. & n.a. & n.a. & n.a. \\
\hline Intercept & n.a. & n.a. & -6.387 & n.a. & n.a. & $\mathrm{P}=0.0028$ \\
\hline Gender & n.a. & n.a. & -0.543 & 0.581 & (0.112 to 3.001$)$ & $\mathrm{P}=0.5200$ \\
\hline Age & n.a. & n.a. & 0.052 & 1.054 & (0.998 to 1.113 ) & $\mathrm{P}=0.0603$ \\
\hline Smoking & n.a. & n.a. & 3.281 & 26.595 & (2.595 to 272.600$)$ & $\mathrm{P}=0.0057$ \\
\hline $\mathrm{mSHOX} 2$ & n.a. & n.a. & 2.396 & 10.979 & (1.999 to 60.300) & $\mathrm{P}=0.0058$ \\
\hline
\end{tabular}




\section{Discussion}

Alterations in DNA methylation are currently one of the most promising biomarkers in clinical research and thus SHOX2 methylation may represent the potential improvement in lung cancer management. Expression of this gene is present in various tumor types, such as neuroblastomas and breast cancer [24].

The overall frequency of $S H O X 2$ methylation in our set was estimated at 63.8\%, however it depends on the proportion of positive and negative samples in the set. Schmidt et al. [24] reported SHOX2 methylation in $67.6 \%$ of analyzed samples. In the set of bronchial lavages the partial frequency of SHOX2 methylation was estimated at the level $57.4 \%$.

The average age of negative patients (54.1 years) and positive patients (66.7 years) reflects the expectation that the non-malignant samples were collected from younger patients than the positive ones. This finding confirms the fact that malignancies are usually associated with older age (Table 1). To accommodate this difference in age distribution the final model was adjusted for age (Table 4).

The smoking status of analyzed group was also monitored and higher percentage of non-smokers in the negative group than in positive group confirms expected strong and significant association between the presence of cancer and smoking status (Table 1). Due to this fact the models were adjusted to smoking status (Table 4).

Methylation of $S H O X 2$ in tumor samples. In the group of samples with lung cancer confirmed by cytology/histology, $92 \%$ of patients were positive for presence of SHOX2 methylation. Only 3 patients diagnosed with adenocarcinoma were without detectable SHOX2 methylation in any of analyzed material (Table 3). Differences in the observed proportions of $m S H O X 2$ positive test results among patients with squamous carcinoma and adenocarcinoma are in agreement with findings by Kneip et al. [21] and Schmidt et al. [24]. However, our detection rates of methylation in the histological subtypes were higher in magnitude. We identified $100 \%$ SHOX2 methylation in SCLC and $100 \%$ in squamous carcinoma specimens versus $67 \%$ in adenocarcinoma specimens $(\mathrm{P}=0.0381)$, whereas Schmidt et al. [24] reported 97\% SHOX2 methylation in SCLC and $82 \%$ in squamous carcinoma specimens versus only $47 \%$ in adenocarcinoma specimens, although, they do not provide an explanation for the difference in the observed proportions. Our overall detection rate of positive patients was $92 \%$ in comparison with $72 \%$ reported by Kneip et al. [21] and $68 \%$ reported by Schmidt et al. [24]. These results indicate that specific type of lung cancer (SCLC and NSCLC squamous type) may be preferentially associated with $\mathrm{SHOX} 2$ methylation. The same phenomenon was also previously described by Schmidt et al. [24] and requires further investigation.

Methylation of $S H O X 2$ in non-tumor samples. Interestingly, in the negative samples group the SHOX2 methylation samples was detected in $50 \%$ of lymphomas, and in $100 \%$ of

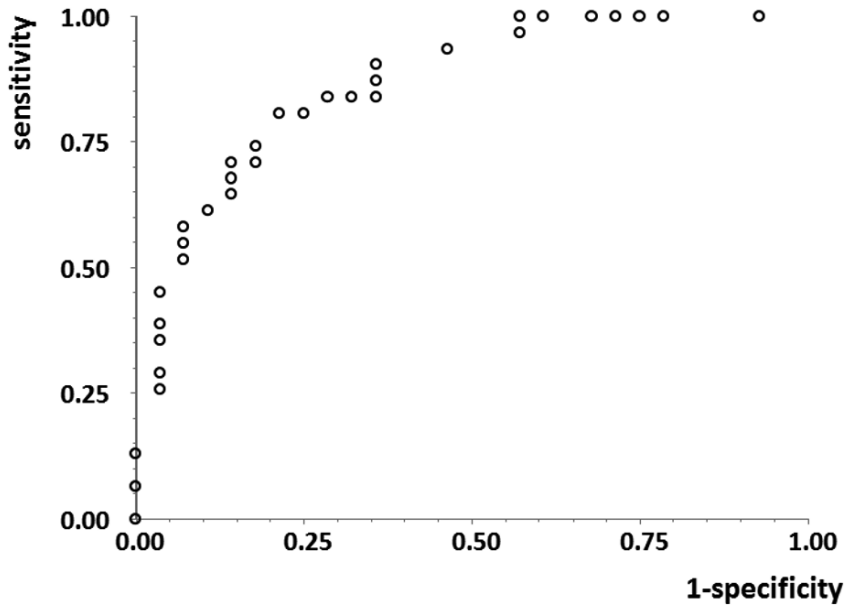

Figure 1. Receiver operating characteristic (ROC) curve for the patient series. Each point on the curve represents the multivariable model performance for a different cut-off probability threshold in deciding whether lung cancer is present.

mesothelioma, pulmonary nodule and metastases samples. This indicates the possibility of using SHOX2 methylation marker in other cancer malignancies as well, which was reported previously by Ilse et al. [25] and Dietrich et al. [26].

Sensitivity and specificity of the test. Overall, 44 patients were detected as positive for SHOX2 DNA methylation in at least one type of material, 9 of these samples were cytologically/ histologically negative. On the other hand, 25 patients were SHOX2 methylation negative and there was no lung tumor found in 3 of these samples. It is to speculate if these samples represented potentially false negative cases (Table 1 ).

These data translate into overall sensitivity of combined assays at $92 \%$ and overall specificity at $71 \%$. Compared to other reported characteristics of $\mathrm{SHOX} 2$ test sensitivities ranging from 60 to $81 \%$ and specificities from 78 to $96 \%$ [20, 21, 23, $25]$ our results are in general concordance. Above average sensitivity may be explained by use of two different materials in each patient.

If we look at each type of material separately, the sensitivity of the assay using bronchial lavage alone was $83.78 \%$ and specificity was $84.62 \%$. Using the combined diagnostic model constructed from bronchial lavage assay, age, gender and smoking status increased sensitivity up to $89.19 \%$ with practically unchanged specificity. Classification performed with cut-off point of 0.5 was correct for $85.71 \%$ patients. Unlike lavage, blood plasma samples are collected non-invasively, thus representing a promising alternative. Similarly as with lavage samples, sensitivity and specificity was settled using logistic regression model at reasonable levels $80.65 \%$ and $78.57 \%$, respectively, using same cut-off 0.5 and with $79.66 \%$ correctly classified patients. Kneip et al. [21] detected comparable sensitivity at $60 \%$ and somewhat higher specificity at $90 \%$. These numbers were certainly affected by many factors such as 
transportation of fresh blood, lower amounts of tumor DNA mainly in early stages of malignant disease as well as type of the normalization controls. Quite a few tests resulted in invalid analysis due to the insufficient amount of DNA isolated. All these issues need to be tuned before the assay can be used in clinics. However, we think that non-invasive character and relatively good sensitivity/specificity of this method at this point still make it a promising clinical choice.

The bronchial lavage showed higher levels of sensitivity and specificity of $S H O X 2$ methylation test compared to blood plasma. Thus we can conclude that performance of analysis at bronchial lavage samples represents the "gold standard" of lung cancer diagnosis using $\mathrm{SHOX} 2$ methylation marker. However, the plasma samples may be a good alternative source of tumor DNA for SHOX2 methylation analysis. Especially for patients who, for any reason, are unable to undergo bronchoscopy.

Agreement of methylation presence in different type of material. The SHOX2 methylation was not correlating between sample types in 13 cytologically positive samples and 7 negative samples. Out of positive samples, 10 were lavage mSHOX2 positive ( $m S H O X 2+)$ and plasma mSHOX2 negative ( $m S H O X 2-)$. This may be explained by inappropriate sample transport, or insufficient amount of free tumor DNA in patient's plasma as discussed above. The case of 3 other samples which were lavage $\mathrm{mSHOX} 2$ - and plasma $\mathrm{mSHOX2+}$ is little difficult to explain. One may speculate about insufficient number of tumor cells in lavage or presence of mucus or other contaminants, which may have affected the outcome of the lavage samples, or also presence of hidden malignant process.

In the group of negative samples, 4 were lavage mSHOX2and plasma mSHOX2+. However, all of these samples came from patients with history of non-lung tumors. One of the patients was diagnosed with lymphoma in the past; others had metastasis of liver cancer, mesothelioma and sarcoidosis respectively. These findings may indicate that in most of these samples the presence of other type of cancer was reported and thus SHOX2 may represent a promising marker for other malignancies as well. Again, the negative cytology in the group with 3 lavage mSHOX2+/plasma mSHOX2- samples is harder to explain. However, raised levels of oncomarkers in 2 of these patients may indicate the presence of so far undiscovered malignant process. The last patient denied the surgery therapy and a pulmonary nodule of unknown origin was diagnosed by $\mathrm{x}$-ray.

In conclusion, we have confirmed that the methylation analysis of SHOX2 gene using bronchial lavage samples represents a reliable test which can be used as an additional marker for lung cancer diagnosis in patients with negative cytology, who are positive by imaging methods or who have peripherally located lung lesions. The blood plasma test may represent usable alternative in cases when no bronchial lavage is available. Interestingly, if both types of materials were collected and analyzed from the patient, more tumors would be identified, as suggested by specificity of the combined approach. Further, the blood plasma test may be used for clinical decision based on a multivariable model where a diagnostic rule is created from multiple tests.

Finally, our results suggest that SHOX2 methylation may be a potential marker for other malignancies. However, further analyses and validations are needed in order to identify these types of malignancies and the value of this test for them.

Acknowledgements: This publication is the result of the project implementation: 'Establishment of competence centre for research and development in the field of molecular medicine'. Supported by the Research \& Development Operational Programme funded by the ERDF (ITMS 26240220071).

\section{References}

[1] MOLINA JR, YANG P, CASSIVI SD, SCHILD SE, ADJEI AA. Non-small cell lung cancer: epidemiology, risk factors, treatment, and survivorship. Mayo Clin Proc. 2008; 83: 584-94. doi: 10.4065/83.5.584. http://dx.doi.org/10.4065/83.5.584

[2] KHUDER SA. Effect of cigarette smoking on major histological types of lung cancer: a meta-analysis. Lung Cancer. 2001; 31: 139-48. http://dx.doi.org/10.1016/S0169-5002(00)00181-1

[3] U.S. Department of Health and Human Services. The Health Consequences of Smoking: A Report of the Surgeon General. Atlanta, GA: U.S. Department of Health and Human Services, Centers for Disease Control and Prevention, National Center for Chronic Disease Prevention and Health Promotion, Office on Smoking and Health, 2004.

[4] PETO R, LOPEZ AD, BOREHAM J, et al. Mortality from Smoking in Developed Countries, 1950-2000: Indirect Estimates from National Vital Statistics. New York, NY: Oxford University Press, 1994.

[5] KAVCOVA E, ONDRUSOVA M, DZIAN A. Karcinom plúc prekročil hranice tretieho milénia - 1. čast', In: Via Practica. Roč. 7, č. 5 (2010), s. 215-221,

[6] TRAVIS WD, TRAVIS LB, DEVESA SS. Lung cancer. Cancer. 1995; 75(1 Suppl): 191-202. Erratum in: Cancer 1995; 75: 2979. http://dx.doi.org/10.1002/1097-0142(19950101)75:1+<191::AID-CNCR2820751307>3.0.CO;2-Y

[7] SCHWARTZ AG, PRYSAK GM, BOCK CH, COTE ML. The molecular epidemiology of lung cancer. Carcinogenesis. 2007; 28: 507-18. Epub 2006 Dec 20. http://dx.doi.org/10.1093/ carcin/bgl253

[8] HOFFMAN PC, MAUER AM, VOKES EE. Lung cancer. Lancet 2000; 355: 479-85. http://dx.doi.org/10.1016/S01406736(00)82038-3

[9] PETO R, DARBY S, DEO H, SILCOCKS P, WHITLEY E, et al. Smoking, smoking cessation, and lung cancer in the UK since 1950: combination of national statistics with two case-control studies. BMJ. 2000; 321: 323-9. http://dx.doi. org/10.1136/bmj.321.7257.323

[10] ROBERTSON KD. DNA methylation and human disease. Nat Rev Genet. 2005 Aug; 6(8): 597-610. http://dx.doi. org/10.1038/nrg1655 
[11] PALMISANO WA, DIVINE KK, SACCOMANNO G, GILLILAND FD, BAYLIN SB, et al. Predicting lung cancer by detecting aberrant promoter methylation in sputum. Cancer Res. 2000; 60: 5954-8.

[12] BALGKOURANIDOU I, LILOGLOU T, LIANIDOU ES. Lung cancer epigenetics: emerging biomarkers. Biomark Med. 2013; 7: 49-58. doi: 10.2217/bmm.12.111. http://dx.doi. org/10.2217/bmm.12.111

[13] BELINSKY SA, KLINGE DM, DEKKER JD, SMITH MW, BOCKLAGE TJ, et al. Gene promoter methylation in plasma and sputum increases with lung cancer risk. Clin Cancer Res. 2005; 11: 6505-11. http://dx.doi.org/10.1158/1078-0432.CCR$\underline{05-0625}$

[14] LENG S, DO K, YINGLING CM, PICCHI MA, WOLF HJ, et al. Defining a gene promoter methylation signature in sputum for lung cancer risk assessment. Clin Cancer Res. 2012; 18: 3387-95. doi: 10.1158/1078-0432.CCR-11-3049. Epub 2012 Apr 17. http://dx.doi.org/10.1158/1078-0432.CCR-11-3049

[15] DIETRICH D, KNEIP C, RAJI O, LILOGLOU T, SEEGEBARTH A, et al. Performance evaluation of the DNA methylation biomarker SHOX2 for the aid in diagnosis of lung cancer based on the analysis of bronchial aspirates. Int J Oncol. 2012; 40: 825-32. doi: 10.3892/ijo.2011.1264. Epub 2011 Nov 16. http://dx.doi.org/10.3892/ijo.2011.1264

[16] SIMKIN M, ABDALLA M, EL-MOGY M, HAJ-AHMAD Y. Differences in the quantity of DNA found in the urine and saliva of smokers versus non-smokers: implications for the timing of epigenetic events. Epigenomics. 2012; 4: 343-52. doi: 10.2217/epi.12.24. http://dx.doi.org/10.2217/epi.12.24

[17] BLASCHKE RJ, MONAGHAN AP, SCHILLER S, SCHECHINGER B, RAO E, et al. SHOT, a SHOX-related homeobox gene, is implicated in craniofacial, brain, heart, and limb development. Proc Natl Acad Sci U S A. 1998; 95: 2406-11. http://dx.doi.org/10.1073/pnas.95.5.2406

[18] BLASCHKE RJ, HAHURIJ ND, KUIJPER S, JUST S, WISSE LJ, et al. Targeted mutation reveals essential functions of the homeodomain transcription factor Shox 2 in sinoatrial and pacemaking development. Circulation. 2007; 115: 1830-8. Epub 2007 Mar 19. http://dx.doi.org/10.1161/ CIRCULATIONAHA.106.637819

[19] SMITH TM, LOZANOFF S, IYYANAR PP, NAZARALI AJ. Molecular signaling along the anterior-posterior axis of early palate development. Front Physiol. 2013; 3: 488. doi: 10.3389/fphys.2012.00488. eCollection 2012. http://dx.doi. org/10.3389/fphys.2012.00488

[20] DIETRICH D, HASINGER O, LIEBENBERG V, FIELD JK, KRISTIANSEN G, SOLTERMANN A. DNA methylation of the homeobox genes PITX2 and SHOX2 predicts outcome in non-small-cell lung cancer patients. Diagn Mol Pathol. 2012; 21: 93-104. doi: 10.1097/PDM.0b013e318240503b. http:// dx.doi.org/10.1097/PDM.0b013e318240503b

[21] KNEIP C, SCHMIDT B, SEEGEBARTH A, WEICKMANN S, FLEISCHHACKER $M$, et al. SHOX2 DNA methylation is a biomarker for the diagnosis of lung cancer in plasma. J Thorac Oncol. 2011; 6: 1632-8. doi: 10.1097/JTO.0b013e318220ef9a. http://dx.doi.org/10.1097/ JTO.0b013e318220ef9a

[22] BEGUM S, BRAIT M, DASGUPTA S, OSTROW KL, ZAHURAK M, et al. An epigenetic marker panel for detection of lung cancer using cell-free serum DNA. Clin Cancer Res. 2011; 17: 4494-503. doi: 10.1158/1078-0432.CCR-10-3436. Epub 2011 May 24. http://dx.doi.org/10.1158/1078-0432. CCR-10-3436

[23] SCHNEIDER KU, DIETRICH D, FLEISCHHACKER M, LESCHBER G, MERK J, et al. Correlation of SHOX2 gene amplification and DNA methylation in lung cancer tumors. BMC Cancer. 2011;11: 102. doi: 10.1186/1471-2407-11-102. http://dx.doi.org/10.1186/1471-2407-11-102

[24] SCHMIDT B, LIEBENBERG V, DIETRICH D, SCHLEGEL T, KNEIP C, et al. SHOX2 DNA methylation is a biomarker for the diagnosis of lung cancer based on bronchial aspirates. BMC Cancer. 2010; 10: 600. doi: 10.1186/1471-2407-10-600. http://dx.doi.org/10.1186/1471-2407-10-600

[25] ILSE P, BIESTERFELD S, POMJANSKI N, FINK C, SCHRAMM M. SHOX2 DNA methylation is a tumour marker in pleural effusions. Cancer Genomics Proteomics. 2013; 10: 217-23.

[26] DIETRICH D, JUNG M, PUETZER S, LEISSE A, HOLMES EE, et al. Diagnostic and prognostic value of SHOX2 and SEPT9 DNA methylation and cytology in benign, paramalignant and malignant pleural effusions. PLoS One. 2013 Dec 27; 8(12): e84225. doi: 10.1371/journal.pone.0084225. eCollection 2013. http://dx.doi.org/10.1371/journal. pone. 0084225 